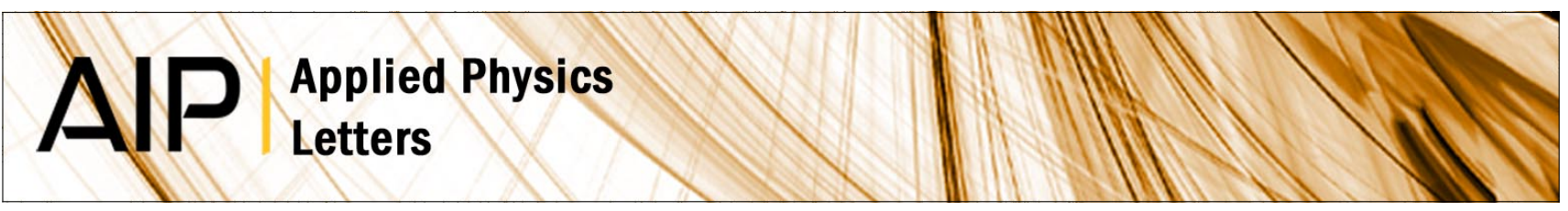

\title{
Imaging crystal orientations in multicrystalline silicon wafers via photoluminescence
}

H. C. Sio, Z. Xiong, T. Trupke, and D. Macdonald

Citation: Appl. Phys. Lett. 101, 082102 (2012); doi: 10.1063/1.4747801

View online: http://dx.doi.org/10.1063/1.4747801

View Table of Contents: http://apl.aip.org/resource/1/APPLAB/v101/i8

Published by the American Institute of Physics.

\section{Related Articles}

Control of the azimuthal orientation of grains in polycrystalline Si films AlP Advances 2, 022153 (2012)

Nanoparticle-mediated nonclassical crystal growth of sodium fluorosilicate nanowires and nanoplates AlP Advances 1, 042165 (2011)

Crystal phase and growth orientation dependence of GaAs nanowires on NixGay seeds via vapor-solid-solid mechanism

Appl. Phys. Lett. 99, 083114 (2011)

Simulation of nucleation in almost hard-sphere colloids: The discrepancy between experiment and simulation persists

J. Chem. Phys. 134, 134901 (2011)

In situ laser crystallization of amorphous silicon: Controlled nanosecond studies in the dynamic transmission electron microscope

Appl. Phys. Lett. 97, 032102 (2010)

\section{Additional information on Appl. Phys. Lett.}

Journal Homepage: http://apl.aip.org/

Journal Information: http://apl.aip.org/about/about_the_journal

Top downloads: http://apl.aip.org/features/most_downloaded

Information for Authors: http://apl.aip.org/authors

\section{ADVERTISEMENT}

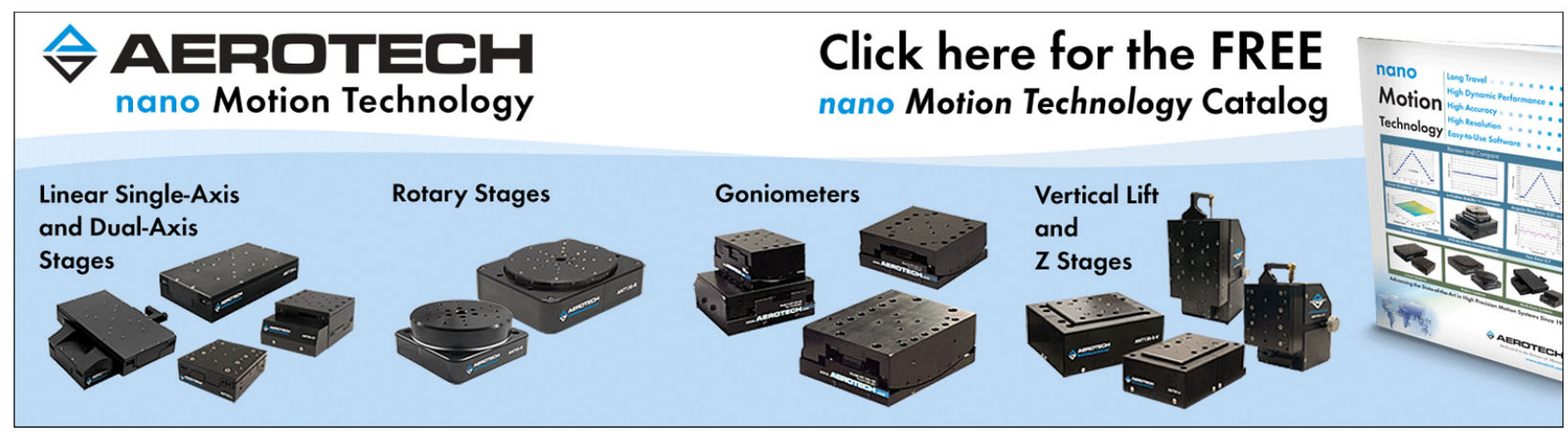




\title{
Imaging crystal orientations in multicrystalline silicon wafers via photoluminescence
}

\author{
H. C. Sio, ${ }^{1, a)}$ Z. Xiong, ${ }^{2}$ T. Trupke, ${ }^{3}$ and D. Macdonald ${ }^{1}$ \\ ${ }^{1}$ Research School of Engineering, The Australian National University (ANU), Canberra, ACT 0200, Australia \\ ${ }^{2}$ State Key Laboratory of PV Science \& Technology, Trina Solar Energy Limited, Changzhou 213031, China \\ ${ }^{3}$ BT Imaging Pty Ltd, Surry Hills, Australia
}

(Received 24 July 2012; accepted 9 August 2012; published online 20 August 2012)

\begin{abstract}
We present a method for monitoring crystal orientations in chemically polished and unpassivated multicrystalline silicon wafers based on band-to-band photoluminescence imaging. The photoluminescence intensity from such wafers is dominated by surface recombination, which is crystal orientation dependent. We demonstrate that a strong correlation exists between the surface energy of different grain orientations, which are modelled based on first principles, and their corresponding photoluminescence intensity. This method may be useful in monitoring mixes of crystal orientations in multicrystalline or so-called "cast monocrystalline" wafers. (C) 2012 American Institute of Physics. [http://dx.doi.org/10.1063/1.4747801]
\end{abstract}

Multicrystalline silicon (mc-Si) is the most commonly used material in solar cell manufacturing because of its relatively low cost and reasonable electronic quality. However, while it is convenient to texture monocrystalline silicon wafers with a (100) surface orientation through alkaline etching, surface texturing in mc-Si is more challenging due to the random nature of the crystal orientations and requires processes such as acidic etching or reactive ion etching, which are either less effective at texturing or are more complex. ${ }^{1}$ It is also reported that the crystal orientation affects the thermal oxidation rate and the effectiveness of surface passivation in silicon. Irene $e t$ al. $^{2}$ studied crystal orientation effects on the thermal oxidation rate and found that the oxidation rate decreases in the order of $R_{(111)}>R_{(110)}>R_{(100)}$. Repo et al. ${ }^{3}$ studied $\mathrm{Al}_{2} \mathrm{O}_{3}$ passivated mc-Si wafers and noticed an inhomogeneity in the effective lifetime after passivation through atomic layer deposition (ALD). While it could be due to bulk defects, as suggested by Repo et al., ${ }^{3}$ another likely explanation is the inhomogeneity of surface passivation caused by variations of the crystal orientations, leading to the changes in surface properties such as the density of dangling bonds.

Measurement of crystal orientation is usually performed through x-ray diffraction or electron microscopy techniques, such as X-ray diffraction topography (XRT) and electron backscatter diffraction (EBSD). ${ }^{4}$ Although these methods allow the measurements or mapping of crystal orientation, they are time consuming and can usually only be applied to a small area. We present an approach to monitor the crystal orientations within a mc-Si wafer based on photoluminescence (PL) imaging. Photoluminescence imaging is a fast, non-destructive and spatially resolved measurement technique which can be applied for the determination of carrier lifetime, ${ }^{5}$ interstitial iron concentrations, ${ }^{6}$ series ${ }^{7}$ and shunts resistances, ${ }^{8}$ among many other applications. ${ }^{9,10}$ Giesecke et $a l^{11}$ applied the PL imaging technique to characterise

\footnotetext{
${ }^{\text {a) }}$ Author to whom correspondence should be addressed. Electronic mail: kelvin.sio@anu.edu.au. Tel.: +61 26197 0145. Fax: +61 261250506.
}

as-cut wafers and reported variations in the PL intensity across different grains, which they attributed to the changes in the reflectance. Our approach is based on PL imaging of polished, unpassivated thin wafers. Photoluminescence from such wafers is dominated by the surface recombination velocity (SRV), which depends on the surface structure of each crystal orientation. This provides the basis for a simple method to determine the mixture of grain orientations in multicrystalline silicon wafers.

The effective lifetime of any semiconductor can be written as

$$
\frac{1}{\tau_{e f f}}=\frac{1}{\tau_{b}}+\frac{1}{\tau_{s}},
$$

where $\tau_{b}$ and $\tau_{s}$ represent the bulk lifetime and surface lifetime, respectively. Since the PL intensity is proportional to the effective lifetime, ${ }^{5}$ the PL intensity $I_{P L}$ can be expressed as

$$
\begin{gathered}
I_{P L} \propto \tau_{b} \quad \text { for } \quad \tau_{s} \gg \tau_{b}, \\
I_{P L} \propto \tau_{s} \text { for } \quad \tau_{b} \gg \tau_{s} .
\end{gathered}
$$

Hence, the PL intensity is proportional to the bulk lifetime in sufficiently well passivated wafers. ${ }^{12,13}$ By contrast, in an unpassivated wafer, provided that the bulk lifetime is much higher than surface lifetime, the PL intensity reflects the SRV of each grain, which in turn depends on their crystal orientation. Different crystal orientations have different surface recombination velocities due to the variations in surface structure, such as the density of dangling bonds, and their interactions with surface passivating films, such as deposited films, or even a native oxide. In an as-cut wafer, the contrast between grains is significantly reduced due to the fact that the deep saw damage limits the differences in the intrinsic surface properties and the effectiveness of surface passivation, causing a very high and almost identical surface recombination velocity on all grains. Polished samples allow for the removal of this deep saw damage and avoid optical effects caused by the changes in reflectance in different 
grains, as opposed to alkaline etched samples that often exhibit varying degrees of texturing on different orientations, which can impact on the PL emission properties. The relatively undamaged polished surface then permits the native oxide to provide some level of passivation, while still ensuring that the effective lifetime is surface limited. This provides suitable conditions for observing variations in crystal orientations.

The PL images in this work were obtained with a BT Imaging LIS-R1 tool, described in detail elsewhere. ${ }^{5,6,14}$ It provides PL images with a pixel size of $23 \mu \mathrm{m}$ when using a zoom lens. An $815 \mathrm{~nm}$ laser is used to generate excess carriers and the resulting band-to-band PL radiating from the wafer is captured by a one megapixel silicon charge-coupled device camera.

The samples used in this work were p-type boron doped mc-Si wafers which came from 25\% (W25), 50\% (W50), and $75 \%$ (W75) from the bottom of a commercially grown directionally solidified mc-Si ingot. While the majority of the data was obtained using the W50 wafer, wafers W25 and W75 were used to test the validity of this approach when using wafers with different doping and bulk lifetimes. Each wafer was diced into smaller pieces, then each piece was polished through chemical etching using $\mathrm{HF}$ acid and $\mathrm{HNO}_{3}$ acid in a ratio of 1:8 for more than 6 min with agitation until a mirror-like surface was achieved. All wafers were dipped in HF solution after etching to remove any residual acidgrown oxide on their surfaces. The thickness of the wafers reduced from $180 \mu \mathrm{m}$ in the as-cut state to around $115 \mu \mathrm{m}$ after the polishing. PL images of each wafer were captured a few days after the polishing to allow enough time for the passivation effect of residual HF to vanish and to allow the formation of native oxide, which provides the desired orientation dependent passivation effect. The crystal orientation of selected grains was measured and mapped by EBSD, taken with a Zeiss UltraPlus analytical FESEM.

The surface energy $\left(E_{s}\right)$ of each orientation was determined based on first-principles, ${ }^{15,16}$

$$
E_{s}=\frac{\left(E_{T}-N E_{b}\right)}{2 A}
$$

where $E_{b}$ and $E_{T}$ correspond to the bulk energy of the silicon lattice and the total energy of an N-layer slab constructed according to each orientation, respectively. $A$ is the surface area of the slab. Both bulk and slab energy were modelled using GULP (Ref. 17) in Materials Studio modelling software.

Figure 1 shows PL images of an as-cut wafer and a mirror polished sister wafer. The scale range is centred around the mean in both images, while the maxima and minima are adjusted to retain a constant contrast ratio. This allows a direct comparison of the differences in contrast of the PL signal among different grains. Note that the average PL intensity is reduced in Figure 1(b) due to the reduction in thickness. Dislocation networks appear as dark lines in Figures $1(a)$ and $1(b)$ as they act as recombination centres for carriers and reduce the local lifetime. No obvious difference of the PL signal among different grains can be observed in the as-cut wafer, as shown in Figure 1(a), while a significant
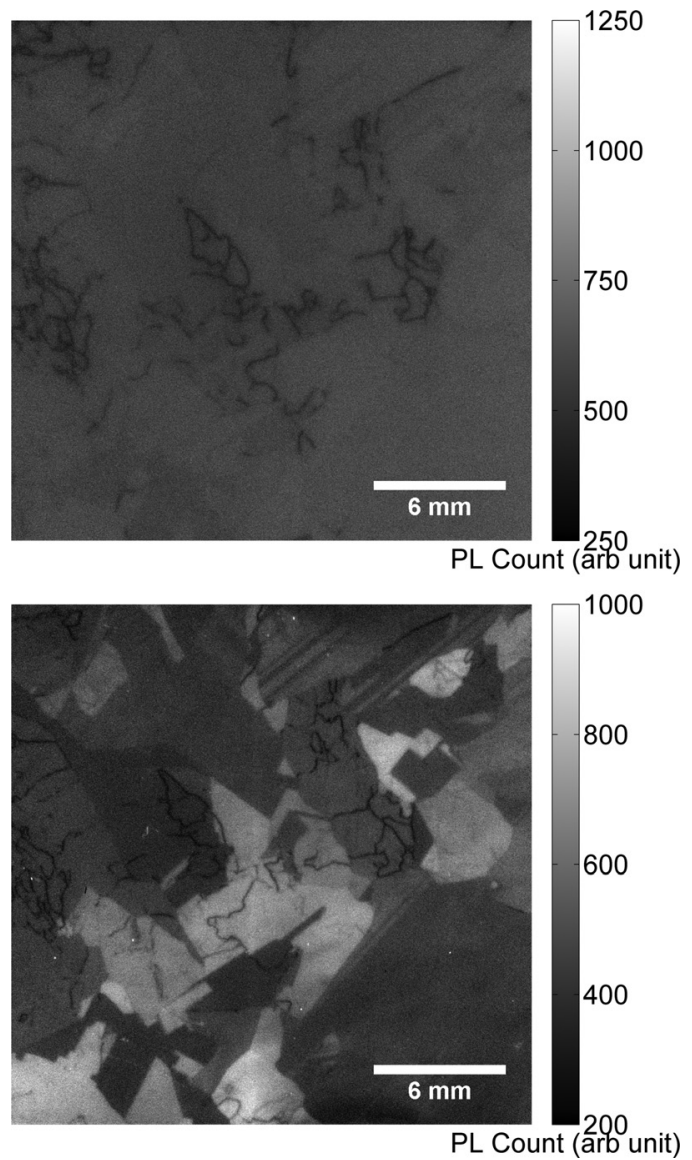

FIG. 1. PL images of (a) as-cut (b) mirror polished wafer, with a pixel size of $23 \mu \mathrm{m}$

contrast among different grains is observed in the mirror polished wafer, as shown in Figure 1(b). The result reflects the fact that different crystal orientations have different SRVs, which are reflected in the PL intensity. Variations of the reflectivity of different grains at $815 \mathrm{~nm}$ in mirror polished wafers were measured to be less than $2 \%$, confirming that the optical properties from grain to grain have no detectible impact on the PL emission.

Figure 2 shows the surface orientation maps, acquired by EBSD, overlayed on the PL image from Figure 1(b). Selected grains in Figure 2(a) are highlighted with different colours according to their surface orientations, in accordance with the inverse pole diagram shown in Figure 2(b). It can be observed that grains with an orientation close to (100) have a higher PL intensity while crystals close to (111) have a lower PL signal.

The surface energy of each detected surface orientation was calculated. Table I compares the modelling result of selected orientations with the results from the literature. Our modelling result agrees well with Jaccodine, ${ }^{18}$ but there is some discrepancy in the surface energy of the (111) orientation between our result and the results from Lu et al. ${ }^{15}$ and Stekolnikov et al. ${ }^{19,20}$ Despite this, the model results are sufficient to demonstrate a definite correlation exists between the PL intensity on mirror polished samples and the surface orientation, which can be represented quantitatively by the surface energy.

Based on the crystal orientation of the detected grains and their corresponding locations in an inverse pole figure 

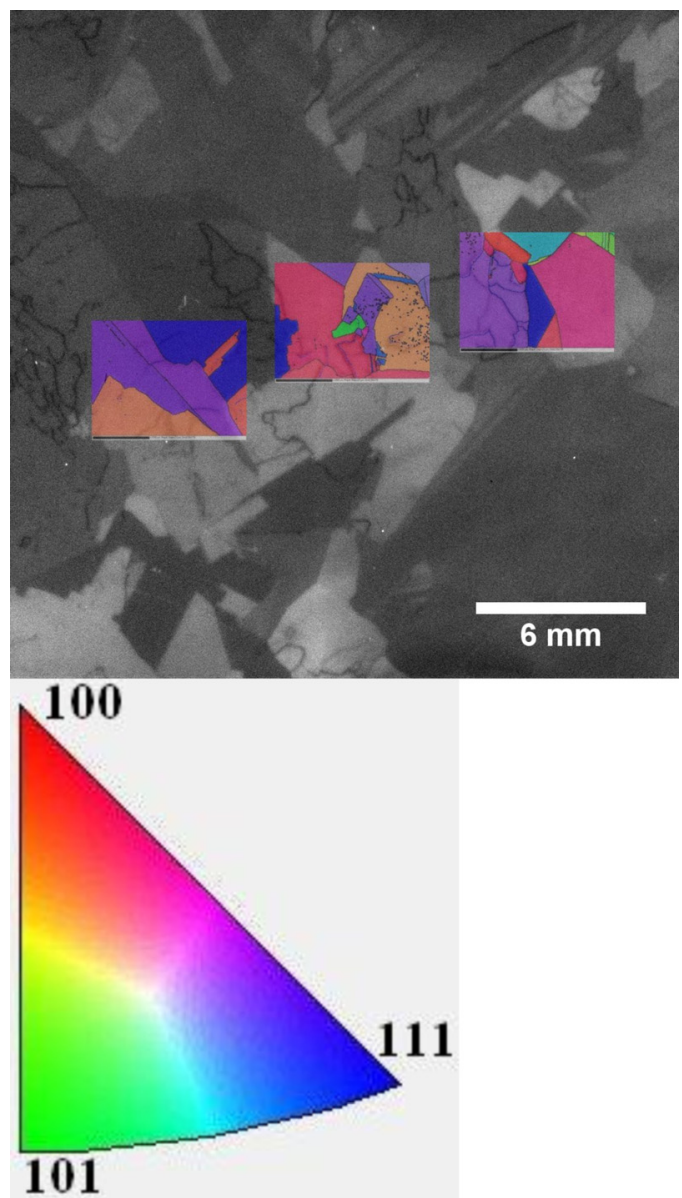

FIG. 2. (a) PL image overlayed with surface orientation. (b) Colour coded inverse pole figure (IPF): represents each surface orientation with a specific colour.

(IPF), we interpolated the surface energy and the PL intensity of various crystal orientations in an IPF using the modelled surface energy and measured PL counts. The result is shown in Figure 3. Figure 3(a) generally agrees with Figure 3 (b), indicating that orientations with a high surface energy show a higher PL signal.

The surface energy can be interpreted as the excess energy at a surface compared with the bulk caused largely by the breaking of atomic bonds at the surface. It is strongly influenced by the density of dangling bonds, which affects the SRV, and it also reflects the difficulty of saturating dangling bonds, which affects passivation effectiveness. We investigated the influence of the surface energy on the PL intensity and the result is shown in Figure 4. W50-A, W50-B, and W50-C correspond to 3 wafers diced from W50. W75

TABLE I. Surface energy comparison between modelling results and literature values.

\begin{tabular}{|c|c|c|c|c|}
\hline \multirow[b]{2}{*}{ Orientation } & \multicolumn{4}{|c|}{ Surface energy $\left(\mathrm{J} / \mathrm{m}^{2}\right)$} \\
\hline & Modelled & Jaccodine $^{18}$ & Lu et al..$^{15}$ & Stekolnikov et al. ${ }^{19,20}$ \\
\hline (100) & 2.26 & 2.13 & 2.37 & 2.39 \\
\hline (111) & 1.19 & 1.23 & 1.99 & 1.74 \\
\hline (113) & 1.95 & & 1.82 & 1.85 \\
\hline (110) & 1.51 & 1.51 & 1.75 & 1.7 \\
\hline
\end{tabular}

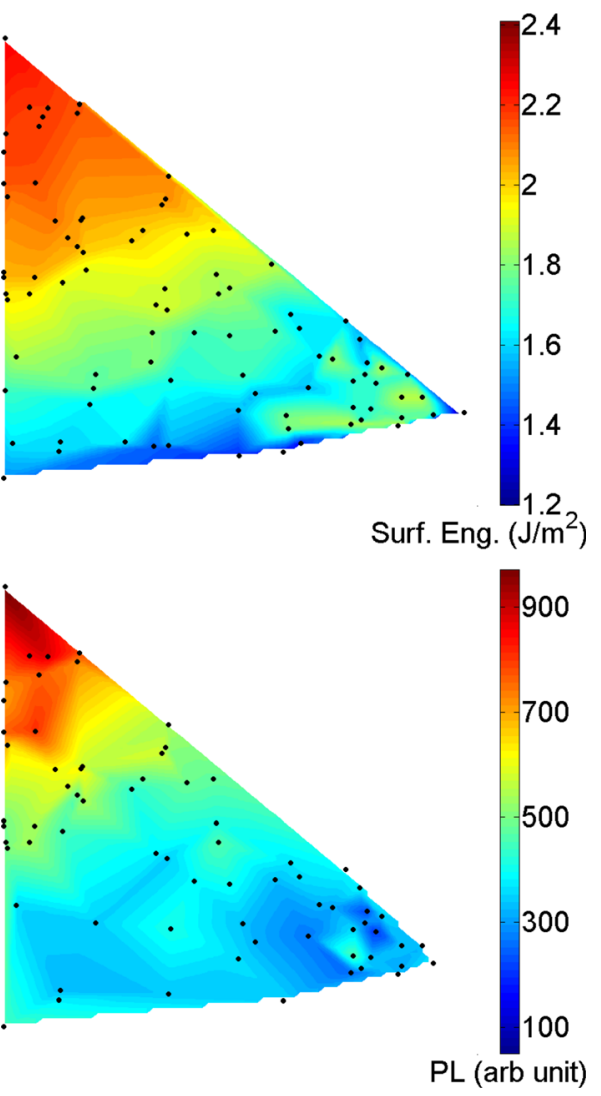

FIG. 3. Interpolated result of (a) surface energy $\left(\mathrm{J} / \mathrm{m}^{2}\right)$. (b) PL count of each orientation plotted in an inverse pole figure (IPF) based on measured grains, represented by dark dots.

and W25 are wafers from $75 \%$ and $25 \%$ from the bottom of the ingot. Due to slight differences in wafer thickness, doping, roughness, and optical properties in the three samples due to the chemical etching, the PL intensity of each grain is normalised using the measured PL intensity of certain grains in W50-A as a reference. Figure 4 shows a strong correlation exists between the PL intensity and the surface energy for all the wafers, including wafers from different parts of an ingot. We suggest that it is possible to estimate the crystal orientation using PL images based on this correlation. While it is difficult to derive an absolute correlation between PL and

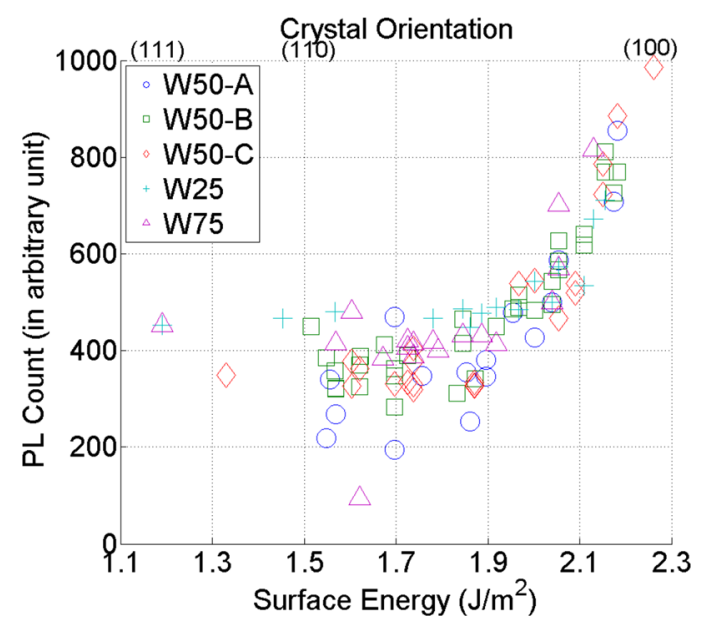

FIG. 4. PL intensity of each detected grain classified according to its corresponding surface energy. 
surface orientation due to the influence of wafer thickness and surface conditions on the measured PL, this method may be useful in estimating mixes of crystal orientations in the border regions of cast monocrystalline wafers, for example, in which the orientation of the large central grain is known, and the corresponding PL intensity can be used for normalisation. Alternatively, in a mc-Si wafer with a large enough number of random grains, the brightest grains can be assumed to be close to (100) orientation. This study also highlights the possibility of using PL imaging to study the influence of crystal orientation on different surface passivation methods in mc-Si wafers, in cases where the impact of the bulk lifetime can be accounted for.

In conclusion, an approach for monitoring crystal orientation based on PL imaging of chemically polished and unpassivated silicon wafers was presented. The PL signal of such wafers is dominated by the surface recombination velocity which is in turn crystal orientation dependent. We observe that grains with an orientation close to (100) have a higher PL intensity while crystals close to (111) have a lower PL signal. Our result also shows a strong correlation exists between surface energy of each orientation and their corresponding PL intensity.

H. C. Sio acknowledges scholarship support from BT Imaging and the Australian Solar Institute, and the Centre for Advanced Microscopy at ANU for SEM access. This work has been supported by the Australian Research Council.
${ }^{1}$ D. H. Macdonald, A. Cuevas, M. J. Kerr, C. Samundsett, D. Ruby, S. Winderbaum, and A. Leo, Sol. Energy 76(1-3), 277 (2004).

${ }^{2}$ E. A. Irene, H. Z. Massoud, and E. Tierney, J. Electrochem. Soc. 133(6), 1253 (1986)

${ }^{3}$ P. Repo, H. Talvitie, S. Li, J. Skarp, and H. Savin, Energy Procedia 8, 681 (2011).

${ }^{4} \mathrm{~V}$. Randle, The Measurement of Grain Boundary Geometry (Institute of Physics, 1993).

${ }^{5}$ T. Trupke, R. A. Bardos, M. C. Schubert, and W. Warta, Appl. Phys. Lett. 89(4), 044107 (2006).

${ }^{6}$ D. Macdonald, J. Tan, and T. Trupke, J. Appl. Phys. 103(7), 073710 (2008).

${ }^{7} \mathrm{H}$. Kampwerth, T. Trupke, J. W. Weber, and Y. Augarten, Appl. Phys. Lett. 93(20), 202102 (2008)

${ }^{8}$ O. Breitenstein, J. Bauer, T. Trupke, and R. A. Bardos, Prog. Photovoltaics 16(4), 325 (2008).

${ }^{9}$ T. Trupke, R. A. Bardos, M. D. Abbott, P. Würfel, E. Pink, Y. Augarten, F. W. Chen, K. Fisher, J. E. Cotter, and M. Kasemann, in Proceeding of the 22nd EPVSC, Milan, Italy (2007).

${ }^{10}$ F. Yan, S. Johnston, K. Zaunbrecher, M. Al-Jassim, O. Sidelkheir, and K. Ounadjela, Phys. Status Solidi (RRL) 6(5), 190 (2012).

${ }^{11}$ J. A. Giesecke, M. The, M. Kasemann, and W. Warta, Prog. Photovoltaics 17(4), 217 (2009).

${ }^{12}$ T. Mchedlidze, W. Seifert, M. Kittler, A. T. Blumenau, B. Birkmann, T. Mono, and M. Muller, J. Appl. Phys. 111(7), 073504 (2012).

${ }^{13}$ R. K. Ahrenkiel, S. W. Johnston, W. K. Metzger, and P. Dippo, J. Electron. Mater. 37(4), 396 (2008).

${ }^{14}$ S. Y. Lim, S. P. Phang, T. Trupke, A. Cuevas, and D. Macdonald, J. Appl. Phys. 110(11), 113712 (2011).

${ }^{15}$ G. H. Lu, M. Huang, M. Cuma, and F. Liu, Surf. Sci. 588(1), 61 (2005).

${ }^{16}$ A. Y. Liu, Y.-C. Fan, and D. Macdonald, Prog. Photovoltaics 19(6), 649 (2011).

${ }^{17}$ J. D. Gale and A. L. Rohl, Mol. Simul. 29(5), 291 (2003).

${ }^{18}$ R. J. Jaccodine, J. Electrochem. Soc. 110(6), 524 (1963).

${ }^{19}$ A. A. Stekolnikov, J. Furthmüller, and F. Bechstedt, Phys. Rev. B 65(11), 115318 (2002).

${ }^{20}$ A. A. Stekolnikov, J. Furthmüller, and F. Bechstedt, Phys. Rev. B 67(19), 195332 (2003). 